\title{
Il Père Brumoy e il teatro greco. Una riflessione critica sulla tragedia classica tra la Querelle des Anciens et des Modernes e l'Illuminismo
}

\section{Filippo Fassina}

\section{(2) OpenEdition \\ 1 Journals}

\section{Edizione digitale}

URL: http://journals.openedition.org/studifrancesi/7799

DOI: ERREUR PDO dans /localdata/www-bin/Core/Core/Db/Db.class.php L.34 : SQLSTATE[HYOOO]

[2006] MySQL server has gone away

ISSN: 2421-5856

\section{Editore}

Rosenberg \& Sellier

\section{Edizione cartacea}

Data di pubblicazione: 1 juillet 2009

Paginazione: 325-333

ISSN: 0039-2944

\section{Notizia bibliografica digitale}

Filippo Fassina, «ll Père Brumoy e il teatro greco. Una riflessione critica sulla tragedia classica tra la Querelle des Anciens et des Modernes e l'Illuminismo», Studi Francesi [Online], 158 (LIII | II) | 2009, online dal 30 novembre 2015, consultato il 08 janvier 2021. URL: http://journals.openedition.org/ studifrancesi/7799 ; DOI: https://doi.org/ERREUR PDO dans /localdata/www-bin/Core/Core/Db/ Db.class.php L.34 : SQLSTATE[HY000] [2006] MySQL server has gone away

\section{(c)}

Studi Francesi è distribuita con Licenza Creative Commons Attribuzione - Non commerciale - Non opere derivate 4.0 Internazionale. 


\section{Il Père Brumoy e il teatro greco. Una riflessione critica sulla tragedia classica tra la Querelle des Anciens et des Modernes e l'Illuminismo}

Il Seicento è il secolo della Querelle des Anciens et des Modernes. Non è qui il caso di riassumere una problematica o di ripercorrere le tappe di un dibattito che caratterizza la cultura europea per un secolo. La bibliografia sul problema è vastissima ${ }^{1}$, anche se troppi testi fondamentali non sono mai più stati né editi né studiati. La recente antologia, a cura di Anne-Marie Lecoq ${ }^{2}$, che copre il periodo dal 1634 (le Questions inouïes di Mersenne) al 1761 (le Querelles littéraires... di Augustin-Simon Irailh), ha in parte colmato questa lacuna. Soprattutto lo straordinario saggio di Marc Fumaroli premesso all'antologia (una vera e propria monografia), Les abeilles et les araignées $^{3}$, ha offerto un'interpretazione acuta e complessiva della Querelle, in quanto fenomeno che fornisce una chiave interpretativa della letteratura dal Barocco al Classicismo illuminista. Il discorso della Querelle riguarda la totalità dei generi letterari, ma privilegia il teatro (che ancora nel Romanticismo sarà al centro delle dispute sulla letteratura).

Nella fase terminale della Querelle, abbiamo un'opera che eserciterà a livello europeo un influsso profondo sulla diffusione e conoscenza della letteratura greca, del teatro in particolare, e che si inserisce nella tradizione di quei parallèles che trovano un modello esemplare nell'opera di Charles Perrault $(1688-1697)^{4}$ : si tratta del Theatre des Grecs ${ }^{5}$, un'impresa monumentale del padre gesuita Pierre Brumoy, interessante figura di erudito e poligrafo ${ }^{6}$.

L'edizione del 1730, in tre tomi, per un insieme di circa millecinquecento pagine, offre una traduzione integrale di tre tragedie di Sofocle (Edipo re, Elettra, Filottete), quattro tragedie di Euripide (Ippolito, Ifigenia in Aulide, Ifigenia in Tauride, Alcesti) e del dramma satiresco Il ciclope, sempre di Euripide. Di tutte le altre tragedie greche, comprese quelle di Eschilo, e delle undici commedie di Aristofane il Père Brumoy appresta corposi riassunti inframmezzati da brani tradotti. Lo scopo è di offrire un repertorio di facile consultazione per gli autori di teatro moderni. La scelta antologica,

(1) Cfr. H. Gillot, La Querelle des Anciens et des Modernes en France, Paris, Champion, 1914 (Genève, Slatkine Reprints, 1968); AA. Vv., La polémique au XVII siècle, «Littératures Classiques», 59 (2006), in particolare pp. 351-365.

(2) Aa. Vv., La Querelle des Anciens et des Modernes, éd. établie et annotée par A.-M. LeCOQ, Paris, Gallimard, 2001.

(3) M. FumAROLI, Les abeilles et les araignées, in AA. Vv., La Querelle des Anciens et des Modernes, cit., pp. 7-220.

(4) Parallele des Anciens et des Modernes, à Paris, chez Jean-Baptiste Coignard, t. I, 1688; ibid., t. II, 1690; chez Jean-Baptiste Coignard Fils et Veuve Jean-Baptiste Coignard, t. III, 1692; ibid., t. IV, 1697.
(5) À Paris, chez Rollin Père, Jean-Baptiste Coignard Fils, Rollin Fils, 1730, 3 tomes (sigla: B).

(6) Per alcune notizie sulla vita e le opere del P. Brumoy, cfr. CH. Daniel, Les jésuites instituteurs de la jeunesse française au XVII et XVIII siècle, $\mathrm{Pa}$ ris-Bruxelles, Société Générale de Librairie Catholique, 1880 , pp. 155-160; C. Sommervogel, Bibliothèque de la Compagnie de Jésus, première partie: Bibliographie (par les Pères Augustin et Aloys DE BACKER), Bruxelles-Paris, Société Belge de Librairie-Librairie des Archives nationales et de l'École des Chartes, 1890-1932, t. II, pp. 243-252; CH. E. O’Neill-J. M. Domínguez (Directores), Diccionario bistórico de la Compañía de Jesús, Roma-Madrid, Institutum Historicum, S. I.-Universidad Pontificia Comillas, 2001, t. I, pp. 560-561. 
stando alle parole dello stesso autore, non sarebbe dettata da motivazioni estetiche o stilistiche, ma da criteri basati sulla maggiore o minore affinità al gusto e allo spirito del teatro moderno. Per esempio, nel caso di Eschilo, Brumoy afferma di non averlo tradotto volutamente, in quanto poco adatto al gusto del pubblico francese e troppo difficoltoso da riprodurre in una lingua diversa dal greco.

Per tutte le pièces tragiche antiche tradotte e per parecchie di quelle riassunte viene inserito un commento e un raffronto con pièces 'moderne' italiane del Cinquecento (l'Edipo tiranno di Orsatto Giustiniani ${ }^{7}$, l'Ifigenia, la Giocasta e la Medea di Lodovico Dolce) e francesi del Seicento (l'Edipe e la Médée di Pierre Corneille, la Phèdre, l'Iphigénie e la Thébaïde di Racine, l'Iphigénie, l'Hercule mourant e l'Antigone di Rotrou) e con Seneca, le cui tragedie sono anche interamente riassunte.

L'importanza dell'opera è dovuta al fatto che la cultura europea, settecentesca e oltre $^{8}$, si servirà della traduzione antologica del Père Brumoy come fonte per la conoscenza del teatro greco. È il caso, per esempio, di Alfieri che legge i tragici greci nella silloge di Brumoy, ben prima di dedicarsi allo studio della lingua greca. Lo stesso tragediografo italiano, infatti, nell'autobiografia, dichiara esplicitamente, per quanto concerne il suo Polinice, dei debiti verso Brumoy'. Anche Voltaire apprezza, malgrado alcune critiche, il Theatre des Grecs, a proposito del quale sottolinea (nel suo Catalogue de la plupart des écrivains français qui ont paru dans le siècle de Louis XIV, pour servir à l'bistoire littéraire de ce temps) che si tratta della migliore opera di questo genere, pur rimproverando a Brumoy di non aver percepito abbastanza la superiorità del teatro francese su quello greco ${ }^{10}$.

I criteri di scelta, le affermazioni sul teatro di Eschilo, come pure la deprecazione che il teatro antico sia caduto nell'oblio, oscurato dalle grandi personalità del teatro moderno, situano Brumoy nel partito degli Anciens all'interno della Querelle che si sta in parte esaurendo.

Alla sezione dedicata al teatro tragico sono premessi un'introduzione generale (Discours sur le Theatre des Grecs), che illustra le intenzioni dell'autore e il piano dell'opera, e due trattatelli (Discours sur l'origine de la tragedie e Discours sur le parallele des theatres), mentre la sezione dedicata alla commedia è preceduta da tre discorsi (un Discours sur la comedie, delle Observations preliminaires e dei Fastes de la guerre $d u$ Peloponnese) e seguita da una Conclusion generale, anch'essa sulla commedia e da un Discours sur le Cyclope d'Euripide, et sur le spectacle satyrique. Mi soffermerò sul secondo dei due Discours relativi alla tragedia, quello Sur le parallele des theatres ${ }^{11}$, di notevole rilevanza teorica.

(7) La pièce di Orsatto Giustiniani, come sottolinea lo stesso Brumoy, è in realtà un volgarizzamento sofocleo.

(8) Il Theatre des Grecs sarà ancora ristampato nel 1826.

(9) Alfieri, riferendosi al Polinice, oltre a un debito nei confronti di Racine, ne riconosce uno nei confronti di Brumoy: «Nel Polinice l'avere io inserito alcuni tratti presi nel Racine, ed altri presi dai Sette Prodi di Eschilo, che legicchiai nella traduzion francese del Padre Brumoy, mi fece far voto in appresso, di non più mai leggere tragedie d'altri prima d'aver fatte le mie [...]» (cfr. V. AlfierI, Vita di Vittorio Alfieri scritta da esso, in ID., Opere, t. I, introduzione e scelta di M. FUBINI, testo e commento a cura di A. Di BENEDETTO, Milano-Napoli, Ricciardi, 1977, pp. 1-403, qui pp. 184-185.
(10) Cfr. Catalogue de la plupart des écrivains français qui ont paru dans le siècle de Louis XIV, pour servir à l'histoire littéraire de ce temps, in VOLTAIRE, Euvres historiques, édition présentée, établie et annotée par R. POMEAU, Paris, Gallimard, 1957 ( «La Pléiade»), pp. 1133-1214, qui p. 1144: «[...] Son Théâtre des Grecs passe pour le meilleur ouvrage qu'on ait en ce genre, malgré ses fautes et l'infidélité de la traduction. Il a prouvé par ses poésies qu'il est bien plus aisé de traduire et de louer les anciens que d'égaler par ses propres productions les grands modernes. On peut d'ailleurs lui reprocher de n'avoir pas assez senti la supériorité du théâtre français sur le grec, et la prodigieuse différence qui se trouve entre le Misanthrope et les Grenouilles». Noteremo che Voltaire attribuisce a Brumoy il nome di Jean.

(11) B, I, pp. xcix-clx. 
In quanto parallèle, appunto, il discorso in questione adotta il metodo della comparaison du moderne avec l'ancien. Così, infatti, Brumoy inaugura la sua trattazione:

On ne fait aucune difficulté de comparer la peinture ou la sculpture moderne avec l'ancienne; ceux même qui excellent aujourd'hui dans l'un ou l'autre de ces arts conviennent sans en rougir, que malgré les efforts des plus sublimes Génies dont les œuvres feront l'admiration de tous les siecles qui les verront, l'antique Grec conserve toujours la superiorité sur ce que nous avons de plus parfait en ce genre. Il n'y a pas deux voix là-dessus: mais il n'en est pas ainsi des ouvrages d'esprit. La comparaison du moderne avec l'ancien semble odieuse à quelquesuns, témeraire à plusieurs, et hardie à ceux qui sans être idolatres de l'antiquité ne laissent pas de la respecter encore. Le goût, qui doit être le souverain juge dans ces deux genres, n'est-il donc pas le même? il l'est sans doute. Mais il va plus sûrement en fait de peinture et de sculpture, étant guidé par les yeux; et plus timidement en matiere d'écrits, où il n'a pour guide qu'une vûë toute spirituelle, qu'une lumiere si épurée, si fine, et si déliée, (s'il est permis de parler ainsi,) que les moindres ombres du préjugé la brouillent sur le champ, et la changent en ténebres. Osons toutefois hazarder l'usage de cette lumiere, et confronter le Théatre ancien avec le moderne, pour atteindre du moins à marquer à peu près l'étenduë et les limites que le goût donne à ce parallele, et pour tirer en faveur de l'un et de l'autre des conséquences si nettes que la partialité ne puisse les désavouer (B, I, pp. xcix-c).

Fin da questa apertura noi ritroviamo alcuni concetti e alcune parole chiave sia della Querelle sia dell'estetica sei-settecentesca. È il caso del termine gô̂t che ritornerà come un motivo conduttore in tutto il discours di Brumoy ${ }^{12}$. Goût, che viene eletto come elemento sovrano di giudizio nella comparaison dei moderni con gli antichi; goût, che si esprime con sicurezza maggiore là dove è questione di arti figurative, quasi a voler riconoscere che pittura e scultura hanno dei canoni estetici di riferimento ormai definiti, che hanno consacrato senza discussione la superiorità dell' antico' greco rispetto alla produzione artistica delle epoche moderne. Non stiamo, ora, a discutere sulla consistenza di tale giudizio - che sarà peraltro al centro dei débats del Neoclassicismo settecentesco - e neppure a chiarire che 1'‘antico', di cui è qui questione, non è tanto il modello iconografico greco, quanto piuttosto il modello rinascimentale (di quel Raffaello in primis, che secondo Berenson avrebbe rivestito col sogno della classicità il nostro immaginario cristiano e moderno $\left.{ }^{13}\right)$. Quello che dobbiamo sottolineare è il fatto che si riconosca una difficoltà e un'incertezza - anche a livello di gôut - nel giudicare gli ouvrages d'esprit (gli scritti letterari), in quanto non la vista materiale, ma una vue toute spirituelle è guida all'interpretazione. Formulazione peraltro fascinosa, ma assai poco critica, che Brumoy in qualche modo spiega, là dove fa riferimento alle ombres du préjugé, evocando attraverso il concetto di 'pregiudizio' le prese di posizione contrastanti dei partiti avversi partecipi della Querelle.

Comunque, se di comparaison si tratta, vediamo rapidamente come viene costruito il riferimento all'‘antico', in una definizione contrastiva del gôut degli antichi e del gồt dei moderni, per quanto concerne limitativamente il discorso sul teatro classico, oggetto degli interessi di Brumoy.

Anzitutto abbiamo, all'interno di un excursus sulla storia greca, l'evocazione del mito di Atene, mondo ideale di civiltà perfetta sia sul piano della vita politica che su

(12) Sulla nozione di bon gô̂t nell'estetica settecentesca cfr. J.-P. SERMAIN, Le code du bon gồt (1725-1750), in AA. Vv., Histoire de la rbétorique dans l'Europe moderne (1450-1950), sous la direction de M. Fumaroli, Paris, P.U.F., 1999, pp. 879943.
(13) Cfr. B. Berenson, The italian Painters of the Renaissance, London, Phaidon Press, 1954 (raccoglie quattro saggi pubblicati separatamente tra il 1894 e il 1907): trad. it. di E. CECCHI, I pittori italiani del Rinascimento, Firenze, Sansoni, 1957, pp. 127-129. 
quello della creazione intellettuale. Viene fra l'altro ripreso il tema, caro a una storiografia secolare, dell'età dell'oro - o meglio del secolo perfetto - in cui ogni popolo giunge à son comble de grandeur, per poi decadere:

[...] et voilà le commencement du siecle le plus brillant d'Athenes, du siecle de la grandeur, de la magnificence, des Richesses; des monumens et des spectacles; du siecle des poëtes, des philosophes, des orateurs, des historiens, des héros, et des grands hommes en tout genre. C'est celui de la Tragédie surtout, et de ses trois Autheurs qui l'élevérent au point où nous la représentons aujourd'hui dans cet ouvrage. Après avoir coulé legerement sur les siecles anterieurs d'Athenes, il me paroît nécessaire d'insister un peu plus sur celui qui fut la source de tant de merveilles, soit en paix, soit en guerre. Il semble que le destin de chaque nation soit d'avoir son bel âge et son comble de grandeur où elle arrive par des progrès insensibles, et dont elle descend ensuite imperceptiblement et par degrés. Tel fut le siecle d'Auguste; et tel a été longtems auparavant celui d'Athenes (B, I, pp. civ-cv).

Questa lettura dell'antichità e questa impostazione storiografica, che troverà nel Settecento la sua maggiore e più illustre sistemazione nel Siècle de Louis XIV di Voltaire, ha in Brumoy un corollario politico, anch'esso debitore di una mitologia storica e forse anticipatore di tematiche che saranno proprie dei Lumi, nella connessione della grandezza culturale con la grandezza civile, sullo sfondo del ricordo delle lotte 'repubblicane' di Atene, in difesa della libertà, contro une vaste Monarchie di tiranni ed oppressori:

Athenes osa compter sur ses forces qui n'étoient rien en comparaison de celles de la Perse et du grand Roi; ainsi nommoit-on le Roi de Perse. Une Republique très bornée eut la hardiesse de porter ses armes dans le sein d'une vaste Monarchie, et mit toute sa politique à empêcher l'ennemi de la pénétrer elle-même. Elle y réussit. Datis Géneral des Perses voulut par represailles entrer bien avant dans l'Attique. Les Atheniens le prévinrent. Ils allerent à sa rencontre. Secondés seulement de ceux de Platée; et conduits par Miltiade ils gagnerent la célebre bataille de Marathon, où se trouva Eschyle aussi grand guerrier que bon poëte. Cette victoire, qui couta la vie à Hippias, 6400 hommes aux ennemis, et moins de deux cens aux Atheniens, enfla extrêmement le cœur de ces peuples redevenus libres et republicains. La terreur qu'elle répandit chés les Perses, les préparatifs de trois années ausquels elle les engagea pour réparer cet échec, l'estime où elle mit Athenes dans toute la Grece et chés les nations voisines, lui inspirerent ces sentimens de grandeur et de fierté dont les Tragédies d'Eschyle sont remplies. Les Atheniens se crurent les arbitres suprêmes de la Grece qu'ils défendoient, et par cette orgueilleuse opinion ils se fraïerent peu à peu une route pour le devenir en effet. Ce fut alors qu'Eschyle nourri dans les idées et dans les exercices de la guerre forma et enfanta la véritable Tragédie, comme nous l'avons expliqué. Ses exemples lui susciterent des rivaux. Mais l'inventeur l'emporta souvent par le succès de l'execution. Tandis qu'il florissoit, on vit naittre Sophocle qui devoit l'imiter et le surpasser. Quinze ans après nâquit Euripide concurrent de ces deux grands Poëtes, et qui a laissé la victoire indécise entre Sophocle et lui. Il vint au monde dix ans après la bataille de Marathon, l'année même que se donna sur mer celle de Salamine, où Leonidas commandoit en chef à la tête des Lacédémoniens tous les alliés Grecs, quoique les Atheniens, sous la conduite de Themistocle, eussent mené la plus grande partie des vaisseaux. Aussi s'en attribuerent-ils tout l'honneur. Cette journée si honteuse pour Xerxès, et si glorieuse pour eux, fut suivie de celle de Platée (B, I, pp. cv-cvi).

A partire da questa evocazione del mito di Atene, mondo di civiltà ideale sul piano politico come su quello intellettuale ${ }^{14}$, si sviluppa il parallèle fra 'antico' e 'moder-

(14) Cfr. anche B, I, p. cxii: «L'abondance et la prosperité y produisent le goût des arts et des sciences. La Tragédie et la Comédie y naissent successivement, et y sont reçûës avec un espéce 
no'. Tutto il discorso comparativo verte sulla rappresentazione di quello che Brumoy definisce caractère sia degli antichi (identificati nel peuple Athénien) sia dei moderni (identificati nei francesi del Grand Siècle). Caractère che egli tenta di individuare mediante la lettura delle pièces tragiche classiche e di quelle francesi del Seicento.

Il fine ultimo del teatro tragico antico sarebbe la celebrazione della polis ateniese: anche quando i personaggi rappresentati dai tragediografi non sono di origine ateniese, sono dotati di un air attique, per poter essere accettati e riconosciuti dalla comunità degli spettatori. In questo confronto, d'altronde, Brumoy ricorda come anche i moderni francesi - l'esempio che egli porta è quello di Corneille - diano voce a personaggi del mito o della storia antica, caratterizzandoli secondo i canoni di comportamento della Francia moderna:

Par le caractere du peuple Athenien, l'on peut marquer celui des Tragédies Grecques. Les Atheniens étoient fous de la liberté, idolatres de leur patrie, adorateurs de leurs usages, dédaigneux ou indifférens pour tout ce qui n'étoit point d'eux. [...] Quels éloges d'Athenes! il n'y a presque pas une piéce de celles qui nous restent où elle ne soit encensée, soit pour l'antiquité de ses monumens, soit pour la sagesse de sa politique, soit pour la prééminence des arts, soit pour la primauté sur le reste de la Grece. [...] Non pas que tous les héros des trois Poëtes soient purement Atheniens, comme on nous a reproché de rendre tous les nôtres François. Ils ne démentent ni leur caractere, ni leur païs. Mais comme ils sont tous tirés de la fable ou de l'histoire Grecque, il a été plus aisé de leur donner un air Attique, sans les déguiser tout-à-fait, qu'il ne l'a été à Corneille de peindre de vieux Romains devant les François, sans leur donner un peu les manieres Françoises, ou de moins un air uniforme: l'air des héros Tragiques de l'antiquité n'est diversifié qu'autant qu'il faut pour les reconnoître (B, I, pp. cxv-cxvi).

Tuttavia, proprio nella connessione fra tragedia e società possiamo riscontrare una differenza sostanziale delle pièces francesi rispetto a quelle antiche. Mentre i tragediografi classici rifiutano categoricamente qualsiasi soggetto e ambientazione che siano estranei alla storia ateniese - o al più greca -, il pubblico francese predilige la rievocazione di mondi remoti, nel tempo e nello spazio. Lo straniero e l'esotico, oggetto di satira nella tragedia e soprattutto nella commedia antica in quanto considerati 'barbari', sembrano invece affascinare l'esprit e il goût dei moderni, condizionandone profondamente la drammaturgia:

D'ailleurs l'amour naturel pour ce qui touche de plus près, portoit les Grecs à n'estimer que ce qui venoit de leurs fonds, bien différens en ceci des François, qui contens d'eux-mêmes pour l'esprit et le goût, préférent ordinairement en fait de plaisir ce qui est étranger et rare à ce qui naît chés eux (B, I, p. cxvii).

Proprio perché coglie queste differenze nell'interpretare i personaggi nel quadro di contesti storici e di sensibilità sociali lontanissime, Brumoy avverte un dato fondamentale nel costruirsi del rapporto antico/moderno proprio di tutti i 'classicismi':

d'idolatrie. Les cérémonies sacrées se changent en divertissemens. L'émulation multiplie les Poëtes, et leur nombre fait établir des disputes, des prix, des couronnes. Le peuple passionné pour les amusemens du Théatre en devient insatiable. Les Théatres s'agrandissent, l'emportent sur les Temples; et toute Athenes se trouve rassemblée dans leur enceinte. On s'infatuë de vers jusqu'à apprendre par cœur les Tragédies entieres, à mesure qu'on les joue; manie, qui devint utile aux soldats faits prisonniers dans la défaite de Sicile. C'étoit assés de sçavoir des vers d'Euripide pour enchanter les Siciliens; ce qui fonda ce proverbe, il est mort en Sicile, où il y récite des vers. Les Rois même des Etats voisins combloient de caresses les bons Poëtes Atheniens, et se croïoient heureux de pouvoir les attirer à leur Cour. Euripide éprouva souvent leurs faveurs; mais la plus flatteuse étoit l'applaudissement d'un peuple aussi éclairé qu'avide de spectacles et de nouveautés. Car ce n'étoit pas seulement la Poësie qui faisoit fortune à Athenes. La Philosophie y tenoit un rang distingué». 
l'impossibilità di penetrare nella loro totalità le allusioni alla realtà contemporanea, realtà che per i lettori o spettatori di epoche diverse appare talvolta incomprensibile. L'acutezza critica di Brumoy individua come legge ineluttabile della creazione artistica questa difficoltà di cogliere l'allusione alla cronaca. Non per nulla, ricorda che le stesse pièces di Corneille e di Racine offrono agli spettatori del Settecento difficoltà di comprensione, là dove sono inequivocabilmente legate ai mours du temps:

Il y a quantité de Sentences dans les Tragédies Grecques dont le sens naturel ne nous frappe plus; mais qui en avoient un très fin, quoi qu'en enveloppé, par l'application qu'en faisoit le parterre qui n'étoit rempli que de bons entendeurs. C'est ce que les Romains n'ont pas compris, eux qui ne firent des Tragédies que pour imiter les Grecs, et pour faire des Tragédies. [...] il en est de ces traits comme de quelques vers de Corneille ou de Racine qu'on sçait avoir été faits par allusion aux mœurs du tems, et qui ne s'entendront plus que dans un sens plus général par la posterité. Si nous ne pouvons rendre raison par tout des allusions Grecques dont je parle, c'est parce qu'on ne les a pas toutes conservées jusqu'à nous, et qu'il seroit ridicule de deviner. Mais il est sensé et il suffit de remarquer que les Grecs étoient extrêmement amateurs de ces allusions, parce que cette observation seule nous porte à ne pas blâmer dans eux ce que nous n'entendons pas, et contribuë à marquer le caractere de leur Tragédie, but unique qu'il faut ici se proposer (B, I, pp. cxxi-cxxii).

Nella costruzione del parallèle, Brumoy vuole evidenziare differenze e analogie fra 'antico' e 'moderno' sempre a partire dal testo tragico. In questo confronto riemergono - sinteticamente e alquanto disordinatamente - alcuni concetti fondamentali di quel dibattito sulla drammaturgia che attraversa tutto il Seicento, e non soltanto i testi della Querelle des Anciens et des Modernes: valgano come esempio le annotazioni sul concetto vraisemblance ${ }^{15}$. A tale proposito, infatti, Brumoy fa alcune considerazioni essenziali sull'impiego del mito e della storia:

Il n'y a que la vrai-semblance dont il puisse être touché. Or il n'est pas vrai-semblable, que des faits aussi grands que ceux de la Tragédie, des faits qui n'arrivent que dans les maisons des Rois, ou dans le sein des Empires, soient absolument inconnus. Si donc le Poëte invente tout son sujet jusqu'aux noms, l'esprit du spectateur se révolte; tout lui paroît incroïable, et la piéce manque son effet faute de vrai-semblance. [...] Outre que le sujet Tragique n'est pas feint chés les Grecs, non-plus que chés-nous, il est tiré de l'histoire ou authorisé par les traditions populaires, qui sont des annales vivantes (B, I, pp. cxxxvi-cxxxvii).

Due sono, comunque, gli elementi di differenziazione su cui Brumoy maggiormente si sofferma. Elementi, peraltro, che servono molto bene a caratterizzare l'idea di classicità propria del Settecento. Anzitutto, Brumoy sottolinea come, per quanto concerne gli ideali di eroismo e regalità - ideali fondatori del genere tragico - si riscontrino profondi mutamenti nei secoli e come la società moderna non possa contare sullo stesso sistema di valori dell'antichità. Riprendendo una discussione che aveva avuto grande spazio all'interno della Querelle, Brumoy ricorda che, se le qualità

(15) Cfr. AbBé D’Aubignac, La pratique du théâtre, éd. par H. BABY, Paris, Champion, 2001, pp. 123 e 126: «[...] en un mot la Vraisemblance est, s'il le faut ainsi dire, l'essence du Poème Dramatique, et sous laquelle il ne se peut rien faire ni rien dire de raisonnable sur la Scène. [...] Il n'y a donc que le Vraisemblable qui puisse raisonnablement fonder, soutenir et terminer un Poème Dramatique: ce n'est pas que les choses véritables et possibles soient bannies du Théâtre; mais elles n'y sont re- çues qu'en tant qu'elles ont de la vraisemblance: de sort que pour les y faire entrer, il faut ôter ou changer toutes les circonstances qui n'ont point ce caractère, et l'imprimer à tout ce qu'on veut représenter». Sulla nozione di vraisemblance, cfr. anche R. RAPIN S. J., Les réflexions sur la poétique de ce temps et sur les ouvrages des poètes anciens et modernes, éd. crit. par E.T. DuBoIs, Genève-Paris, Droz-Minard, 1970, passim. 
morali dell'eroe antico erano indissolubilmente legate a quelle fisiche, secondo un canone di kalokagathia che affondava le radici nelle origini stesse del pensiero occidentale $^{16}$, l'eroe moderno invece si caratterizza come tale per motivazioni esclusivamente morali e si rivela eroe della parola più che della forza:

Ce sont des héros et des Rois de part et d'autre: mais les idées de l'héroïsme et de la Roïauté ont si fort changé, qu'Agamemnon et Achille, l'un Roi des Rois, et l'autre héros des héros, (s'il est permis d'user cette expression,) ne sont plus les mêmes hommes dans Euripide et dans Racine, quoique le fonds de leur caractere soit le même; et il a fallu sans doute que cela fût ainsi, parce que le point de vûë et les yeux étant tout différens, les objets ont aussi dû l'être. [...] Les uns [les anciens] ne connoissent de héros que des hommes distingués du vulgaire par les qualités personnelles autant du corps que du cœur, par la force et la taille autant que par la valeur et la prudence. Les autres [les modernes] accoutumés à une espéce de bravoure plus fine, regardent les héros par les sentimens et par les paroles beaucoup plus que par les effets (B, I, pp.cxl-cxli).

In secondo luogo, la caratterizzazione eroico-politica dei soggetti e dei personaggi della tragedia secentesca si distingue da quella dei soggetti della tragedia classica per l'importanza centrale che occupano amore e galanterie. Se il goût degli spettatori ateniesi escludeva il sentimento amoroso e, in particolare, ciò che i moderni francesi definiranno tendresse, il pubblico francese esige dai tragediografi questi risvolti sentimentali:

Une autre différence très-considérable prise entierement du côté des spectateurs, c'est la galanterie et l'amour. Il n'y en a presque point chés nos Poëtes Grecs. Les spectateurs plus politiques et plus ambitieux que tendres et galans, s'en seroient choqués comme d'une foiblesse indigne de la majesté du Théatre Tragique. Le renversement des Etats, la splendeur des Republiques, le jeu des grandes passions, étoient pour eux des objets conformes à leur caractere orgueilleux et fier, quoique poli. La politesse Françoise devenuë moins fiere et moins ambitieuse dans l'Etat florissant du gouvernement Monarchique, s'est fait par habitude un goût tout contraire, que les faiseurs de spectacles et de Romans ont eu grand soin d'entretenir par leur attention à gagner les suffrages des souveraines arbitres du goût. Les spectatrices Atheniennes n'étoient pas celles qui donnoient la vogue. Différence si marquée, que l'amour occupe souvent les trois quarts des Tragédies Françoises, au lieu que les Grecques se soutiennent d'un bout à l'autre par la seule force de l'action qui en est le fonds (B, I, cxlvi-cxlvii).

Tendresse e galanterie diventano elemento di differenziazione rispetto al mondo classico in una prospettiva che Brumoy vuole fondamentalmente 'politica': infatti, la politesse, supporto di entrambe, fiorisce nella Monarchia francese, che, in questo caso, è quella del Grand Siècle di Louis XIV ${ }^{17}$. D'altronde, due anni prima la pub-

(16) Per l'uso delle formule kalokagathós e kalokagathía vedi Platone, Resp., VI, 5, 489e, e ARIStOtele, Eth. Eud., VIII, 3, 1248b. Cfr. anche W. JAEger, Paideia. Die Formung des griechischen Menschen, Berlin u. Leipzig, Walter de Gruyter \& Co., $1954^{3}$ (trad. it. di L. Emery, Paideia. La formazione dell'uomo greco, Firenze, La Nuova Italia, 1963. 1964, passim e, in particolare, vol. I, pp. 25-48); M. Pohlenz, Der hellenische Mensch, Göttingen, Vandenhoeck \& Ruprecht, 1947 (trad. it. di B. РRото, L'uomo greco, Firenze, La Nuova Italia, 1962, pp. 495-529).

(17) Sulle nozioni di politesse, tendresse e galanterie, cfr. almeno M. MAGENDIE, La politesse mon- daine et les théories de l'honnêteté en France au XVII siècle de 1600 à 1660, Paris, P.U.F., 1925 (rist. anast. Genève, Slatkine, 1970); J.-M. PELous, Amour précieux, amour galant (1654-1675), Paris, Klincksieck, 1980; E. BurY, Littérature et politesse. L'invention de l'bonnête homme (1580-1750), Paris, P.U.F., 1996; M. Daumas, La tendresse amoureuse (XVI'XVIII siècle), Paris, Libraire Académique Perrin, 1996; cfr. anche, per ulteriore bibliografia, AA. VV., La politesse amoureuse de Marsile Ficin à Madeleine de Scudéry. Idées, codes, représentations, Actes du Colloque International de Reims 17-19 novembre 1999, sous la direction de F. GREINER et J.-Cl. TERNAUX, «Franco-Italica», 15-16, 1999. 
blicazione del Theatre des Grecs di Brumoy, nel 1728, anche Luigi Riccoboni in una Dissertation sur la tragedie moderne ${ }^{18}$ - in un parallèle peraltro non fra tragedia antica e tragedia francese, ma fra tragedia italiana e tragedia francese - sottolineava galanterie, politesse e tendresse quali componenti del discorso tragico francese a partire da Corneille ${ }^{19}$, restaurateur e inventeur de la tragédie françoise. Il fatto che, a differenza di Brumoy, Riccoboni veda in questo aspetto un elemento riduttivo non toglie la significativa consonanza dei due critici:

On peut donc appeler Monsieur Corneille le Restaurateur du Théâtre François, bien plus, on peut le nommer l'inventeur de la Tragedie Françoise, parce que la Tragedie de Pierre Corneille, de Thomas son Frere, de Racine, et de tous les autres, qui sont venus après, ne ressemble ni à la Grecque, ni à la Latine, ni à l'Italienne, ni à l'ancienne Tragedie Françoise. Ces habiles Gens vivoient dans un tems, où la Cour de France étoit le modele de la galanterie, et de la parfaite politesse. Ils imaginerent de moderer la severité de la Tragedie, pour rendre ce Spectacle plus convenable à la Cour, et au jeune Roi, et pour cela ils firent l'Amour le maitre, et le souverain du Théâtre. Je ne crois pas qu'on s'éloignât du vrai, si l'on disoit que la Tragedie Françoise est la fille aînée des Romans, puisque le genie Romanesque y domine: ce goût de l'amour Romanesque étoit si fort en vogue dans la Tragedie, que Thomas Corneille dans son Timocrate, n'a fait que copier l'action du Caloandre. L'Amour en peu de tems devint le Tiran du Théâtre, et les Auteurs tragiques l'ont fait entrer dans les sujets, qui non seulement n'en étoient pas susceptibles, mais, si je ne me trompe, qui ne pouvoient pas l'admettre. Monsieur Corneille en transportant sur le Théâtre François l'Oedipe de Sophocle en a alteré l'original, et à la place de Creon, il y a mis Thesee, et y a ajoûté une Princesse, pour avoir la commodité d'y inserer des Scenes de tendresse. Je suis sur qu'avant Monsieur Corneille personne n'auroit jamais crû que l'on pût parler d'amour dans l'Oedipe de Sophocle ${ }^{20}$.

Al di là del raffronto che mette in luce le differenze e le peculiarità della tragedia antica e della tragedia moderna, Brumoy teorizza un concetto di universalità (caractère universel) della letteratura e dell'arte. Un raffronto fra 'antico' e 'moderno' è possibile proprio in virtù del fatto che esiste un elemento generale, che accomuna tutti gli individui, e uno particolare, che li differenzia sia dal punto di vista temporale sia da quello spaziale. Partendo da questa considerazione, lo scopo di ogni opera teatrale risulta quello di divertire e istruire un pubblico costituito da uomini inseriti nel proprio contesto storico-geografico e cioè legati a un goût che è conseguenza diretta del caractère 'nazionale'. Sulla base di questa universalità, anche le modalità per assecondare questo gôut sono le medesime per gli antichi e per i moderni; da qui un'affinità di soggetto, di personaggi e di struttura delle opere tragiche, che permettono una comparaison ancor più approfondita, pur nelle difficoltà insite in un confronto tra mondi concettuali diversissimi e lontanissimi nel tempo:

(18) À Paris, de l'Imprimerie de Pierre Delormel, 1728. Questa Dissertation sarà ripubblicata da Riccoboni nella sua Histoire du Theatre Italien, depuis la decadence de la Comedie Latine [...], par Louis Ricoboni, à Paris, chez André Cailleau, 1730, pp. 247-319.

(19) Ricordiamo che già Corneille, nell'Examen al suo Cdipe, sottolineava necessario immettere nel contesto della storia antica la vicenda amorosa: «[...] et qu'enfin l'amour n'ayant point de part en cette tragédie, elle était dénuée des principaux agréments qui sont en possession de gagner la voix publique» (P. CORNEILLE, Euvres complètes, par G.
Couton, Paris, Gallimard, 1980-1987, «La Pléiade», t. III, p. 20).

(20) L. RICCOBONI, Histoire du Theatre Italien, cit., pp. 267-269. Cfr. su questo testo P. TRIVERO, Luigi Riccoboni, detto Lelio, non solo attore, «Franco-Italica», 1 (1992), pp. 101-118, qui pp. 108-110. All'interno della suddetta Dissertation di Riccoboni, un intero capitolo (Chapitre Troisième. Des effets que produit l'amour dans la Tragedie Françoise. Du retranchement des Choeurs, et des Confidens introduits, in Histoire du Theatre Italien, cit., pp. 271-277) è consacrato al tema della galanterie e dell'amour. 
Faisons d'abord attention que les hommes contemporaines et citoïens du même païs ont dans leur caractere quelque chose de général qui s'étend à tous, et quelque chose de personnel qui les distingue entr'eux. On reconnoît un Italien, un Anglois, un Espagnol, un François d'un coup d'œil. Tous marchent, tous pensent, tous agissent. Mais ils n'agissent, ni ne pensent, ni ne marchent du même air. La différence saute aux yeux. Une différence plus fine et moins apperçûë est celle qui se trouve dans chaque homme de la même nation. Car le caractere universel se sous-divise presqu'à l'infini; et plus cette division est étenduë, plus a-t'on de peine à la déchiffrer. Le livre immortel de la Bruïere, nos bonnes fables, et nos meilleures Comédies ne sont que des ébauches de ces chiffres nombreux qui caracterisent les hommes d'un même climat. Il en est de même des ouvrages poëtiques. Eschyle, Sophocle, et Euripide ont un air Athenien, sans se ressembler. Corneille et Racine ont la physionomie Françoise, sans aucun autre rapport. Il y a plus. Car les Théatres de la Grece, de l'ancienne Rome, de l'Italie moderne, de l'Espagne, et de l'Angleterre, et de la France ont quelque chose de commun; mais ils ont en même tems des différences si marquées, qu'une seule scene suffit pour les faire sentir aux moins connoisseurs, même en supprimant le nom du païs. Le terroir se fait d'abord reconnoître au fruit. Il y a un tour d'esprit qui frappe aussi vivement l'imagination qu'un accent étranger frappe l'oreille. Or c'est, eu égard à ces différences, que le parallele devient difficile. On peut le porter jusqu'à un certain point au-delà duquel le fil de la comparaison se perd. C'est qu'il y a une régle fixe, et une régle arbitraire, dont l'une est inséparable de l'autre quand il s'agit de comparer le moderne avec l'ancien. Presque toute comparaison a ce défaut; mais particulierement celle dont nous parlons, dans laquelle le goût universel n'est le souverain juge que jusqu'aux limites, où le goût arbitraire commence son empire avec un Despotisme qui empiette le plus souvent sur la jurisdiction du premier. Entrons dans le détail, et déterminons autant qu'il est possible les bornes de ces deux goûts. XIII. Les Poëtes, ainsi que les nôtres, avoient à divertir et à instruire des hommes raisonnables par un spectacle majestueux; car il ne faut considerer d'abord les spectateurs que comme des hommes. Les anciens et les modernes s'y sont pris par les mêmes voïes générales pour leur plaire. Même but, même sujets, même œconomie pour le fonds: c'est-à-dire, dessein d'émouvoir une agréable tristesse, sujets grands et nobles de part et d'autre, œconomie réguliere selon l'idée de régularité que chacun s'est formée (B, I, pp. cxxxiii-cxxxv).

Quello che a noi importa, per ora, è avere riassunto brevemente un discorso critico che, sebbene profondamente ancorato alla grande Querelle secentesca, offre uno schema di lettura e di interpretazione, considerato utilizzabile fino alle soglie dell'Ottocento. 